\title{
Modified limited-memory Broyden-Fletcher-Goldfarb-Shanno algorithm for unconstrained optimization problem
}

\author{
Muna M. M. Ali \\ Department of Mathematics, College of Computers Sciences and Mathematics, Mosul University, Iraq
}

\begin{tabular}{l} 
Article Info \\
\hline Article history: \\
Received Dec 6, 2020 \\
Revised Sep 17, 2021 \\
Accepted Sep 21, 2021 \\
\hline
\end{tabular}

\section{Keywords:}

BFGS algorithm global convergence property

Nonmonotone line search

Self-scaling

Unconstrained optimization

\begin{abstract}
The use of the self-scaling Broyden-Fletcher-Goldfarb-Shanno (BFGS) method is very efficient for the resolution of large-scale optimization problems, in this paper, we present a new algorithm and modified the selfscaling BFGS algorithm. Also, based on noticeable non-monotone line search properties, we discovered and employed a new non-monotone idea. Thereafter first, an updated formula is exhorted to the convergent Hessian matrix and we have achieved the secant condition, second, we established the global convergence properties of the algorithm under some mild conditions and the objective function is not convexity hypothesis. A promising behavior is achieved and the numerical results are also reported of the new algorithm.
\end{abstract}

This is an open access article under the CC BY-SA license.

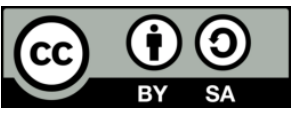

\section{Corresponding Author:}

Muna M. M. Ali

Department of Mathematics

College of Computers Sciences and Mathematics

Mosul University, Al-Majmoaa Street, Mosul, Iraq

Email: munamoh74@uomosul.edu.iq

\section{INTRODUCTION}

Consider the unconstrained optimization problem:

$$
\min _{x \in R^{n}} f_{(x)}
$$

where $f: \mid R^{n} \rightarrow R$ is a continuously differentiable function, to solve problem (1) one uses an algorithm that generates a sequence of iterates $x_{k}$ according to:

$$
x_{k+1}=x_{k}+\alpha_{k} d_{k}
$$

for $k \geq 0$, where $d_{k}$ is a search direction, $\alpha_{k}>0$ is step length and $x_{0}$ is given the initial point. Basic steps in these algorithms are choosing suitable direction and timely step size. To satisfy the descent condition $\nabla f\left(x_{k}\right)^{T} d_{k}<0$, generally, in order to securities a sufficient reduction to value of function we required the search direction $d_{k}$ and $\alpha_{k}$ is specified, there are various examples for procedures to choose the search direction $d_{k}$, conjugate gradient (CG), steepest descent (SD), Newton, quasi-Newton, and trust-region methods see [1]. Newton has the highest rate of convergence and the direction is accounted by solving the system $G_{k} d_{k}=-g_{k}$ where $G_{k}=\nabla^{2} f\left(x_{k}\right)$ and $g_{k}=\nabla f\left(x_{k}\right)$.

Quasi-Newton criterion methods convention the following secant equation: $B_{k+1} s_{k}=y_{k}$ where $y_{k}=g_{k+1}-g_{k}, s_{k}=x_{k+1}-x_{k}$, at the first iteration, $B_{0}$ is an arbitrary nonsingular positive definite 
matrix and $B_{k+1}$ is an approximation of $G_{k}$. The most efficient of Quas-Newton methods are perhaps to selfscaling BFGS method which was updated suggested by [2], [3] and this method is overall numerical computation than the other method. The matrix $B_{k+1}$ in the self-scaling BFGS method can be updated by the following formula:

$$
B_{k+1}=\left[B_{k}-\frac{B_{k} S_{k} S_{k}{ }^{T} B_{k}}{S_{k}{ }^{T} B_{k} S_{k}}\right] \mu_{k}+\frac{y_{k} y_{k}{ }^{T}}{S_{k}{ }^{T} y_{k}}
$$

where:

$$
\mu_{\mathrm{k}}=\mathrm{s}_{\mathrm{k}}^{\mathrm{T}} \mathrm{y}_{\mathrm{k}} / \mathrm{y}_{\mathrm{k}} \mathrm{y}_{\mathrm{k}}^{\mathrm{T}}
$$

If the curvature condition $s_{k}{ }^{T} y_{k}>0$ holds, the method of self-scaling BFGS maintains the positiveness of the matrices $\left\{B_{k}\right\}$. For this reason, the descent direction of $\mathrm{f}$ at $x_{k}$ is satisfy in the direction of the self-scaling BFGS not problem if $G_{k}$ is positive definite or not. Many modifications have been proposed made to afflicted the global convergence property of the (Broyden-Fletcher-Goldfarb-Shanno) BFGS method, for instance, some modulations in the criterion BFGS method are made, and submitteded a modified BFGS (MBFGS) algorithms [4]-[6]. The superlinear convergence and the global of their methods have been proved under appropriate conditions for non-convex problems.

A sufficient reduction produces from suitable line search is another making a good iterative process in function value, as we say. A public situation to accept a step length mentionsed Armijo rule as (5):

$$
f\left(x_{k}+\alpha_{k} d_{k}\right) \leq f_{k}+\sigma \alpha_{k} g_{k}^{T}
$$

and the largest member $\alpha_{k}$ in $\left\{1, \rho, \rho^{2}, \ldots \ldots\right\}$ satisfying (4) such that $\rho \in(0,1)$ and $\sigma \in(0,1)$.

It is clear that $f_{k}$ denotes $f\left(x_{k}\right)$ and $f_{k+1}<f_{k}$ for every descent direction, and called monotone line search. The first non-monotone line search technique were proposed by [7], Newton's method using the Armijo condition was defined by (6):

$$
f\left(x_{k}+\alpha_{k} d_{k}\right) \leq \underset{0 \leq j \leq m(k)}{\max }\left\{f_{k-j}\right\}+\sigma \alpha_{k} g_{k}^{T} d_{k}
$$

where $0 \leq m(k) \leq \min \{m(k+1)+1, N\}, \mathrm{N}$ is a non-negative integer constant, Many kinds of researchers, for example [8]-[12]. A non-monotone schema can promote of finding a global optimum and also developed a speed of convergence. One of the efficient non-monotone line search methods have been proposed by [13] to overcome some drawbacks in the non-monotone in (6) though have features and well work for many situations [14], and have the same general planner while the statement "max" is substitute average weights for values of function with sequential iterations.

\section{MODIFIED A NEW NON-MONOTONE SELF-SCALING BFGS METHOD}

A non-monotone BFGS methods were proposed for solving (1) in [15]-[17]. These algorithms were proved the convergence analysis under the convex hypothesis on the objective function. In this work, a new non-monotone modified self-scaling BFGS method is inserted and evidence the global convergence of the method without convexity assumption. This work is arranged as follows. The New1 non-monotone proposed and defined in line search (7)-(9) and we note that the numerical results of the New1 non-monotone line search (7)-(9) have been more effective than the [18]. The New2 method is expressed in this part. Also, we remember the properties convergence of the new algorithm in part 3. Numerical experiences show that the new method is very favorable and investigated both theatrically and numerically against some well-known algorithms. In the last part, some conclusions are list.

Now we explain the new non-monotone line search method (New1) which is described as follows:

$$
f\left(x_{k}+\alpha_{k} d_{k}\right)-\underset{0 \leq j \leq m(k)}{\max }\left\{f_{k-j}\right\} \leq E_{k}-\sigma t_{k}\left\|g_{k}\right\|^{2}
$$

where:

$$
E_{k}=\delta_{1} t_{k} \delta_{k}^{T} g_{k}
$$




$$
\begin{aligned}
& t_{k}=\frac{\delta_{k-1}{ }^{T} \delta_{k-1}}{\delta_{k-1}{ }^{T} y_{k-1}} \\
& \delta_{1}=0.0001, k \geq 1, \text { with } \sigma \in(0,1)
\end{aligned}
$$

Two reasons made the BFGS algorithm had important disadvantages despite this method is a successful algorithm for unconstrained nonlinear optimization. Once, the directions of the method may not be descent especially when $s_{k}{ }^{T} y_{k}>0$ is not satisfied and cannot guarantee positive definiteness of the matrix $B_{k}$. Second, in general issuess, The BFGS method may not be convergent for non-convex objective functions, despite established superlinear convergence and the global for convex problems.

A New2 non-monotone modified self-scaling BFGS algorithm is presented guaranteeing the positive definiteness of the matrix $B_{k}$ for non-convex objective functions. In this part, the new method is inserted after describing some inspiration. We defined the modified secant equations:

$$
B_{k+1} s_{k}=y_{k}^{*}
$$

where:

$$
y_{k}^{*} \triangleq y_{k}+u_{k}^{*} s_{k}
$$

and defined by three forms:

$$
\begin{aligned}
& u_{k}{ }^{*(1)}=2 \frac{\left\|y_{k}{ }^{*}\right\|^{2}}{S_{k}{ }^{T} y_{k}{ }^{*}} \\
& u_{k}{ }^{*(2)}=1+2 \frac{\left\|y_{k}{ }^{*}\right\|^{2}}{y_{k}{ }^{* T} S_{k}} \\
& u_{k}^{*(3)}=\left\|g_{k}\right\|^{\beta}+\max \left\{\frac{\left\|y_{k}{ }^{*}\right\|^{2}}{y_{k}{ }^{* T} S_{k}}, 0\right\} \geq 0
\end{aligned}
$$

where $\beta$ is a positive constant, see [19], [20]. Then we have reformed the self-scaling BFGS update formula based on (10) as follows:

$$
B_{k+1}=\left[B_{k}-\frac{B_{k} S_{k} S_{k}{ }^{T} B_{k}}{S_{k}{ }^{T} B_{k} S_{k}}\right] \mu *_{k}+\frac{y_{k}{ }^{*} y_{k}{ }^{* T}}{S_{k}{ }^{T} y_{k}{ }^{*}}
$$

where:

$$
\mu *_{k}=s_{k}{ }^{T} y_{k}^{*} / y_{k}^{*} y_{k}^{* T}
$$

and defined an efficient algorithm that is called modified self-scaling BFGS. It is clear that (17).

$$
\left\|g_{k}\right\|^{\beta}\left\|y_{k}{ }^{*}\right\|^{2} \geq y_{k}^{* T} s_{k}>0, \text { for all } k \in N
$$

This property is guarantees positive definiteness of the matrix $B_{k}$ and separate on the convexity of $\mathrm{f}$, as such the used line search. The new MBFGS method combined with the new non-monotone line search and satisfies the global convergence. For unconstrained optimization in which $B_{k}$ is updated in [21], proposed the relation:

$$
B_{k+1}=B_{k}-\frac{B_{k} s_{k} s_{k}{ }^{T} B_{k}}{s_{k}{ }^{T} B_{k} s_{k}}+\tilde{u}_{k} \frac{y_{k}\left(y_{k}\right)^{T}}{s_{k}^{T} y_{k}}
$$

and:

$$
\tilde{u}_{k}=\frac{2}{s_{k}^{T} y_{k}}\left(f_{k}-f_{k+1}+s_{k}^{T} g_{k+1}\right)
$$

so, the local super linear convergence and global properties for convex objective functions preserves in this algorithm too. 
Now, the New2 algorithm is suggested which the self-scaling BFGS method update formula using $y_{k}{ }^{*}$ in (11), and compute the update formula as follows:

$$
B_{k+1}=\left[B_{k}-\frac{B_{k} s_{k} s_{k}{ }^{T} B_{k}}{s_{k} B_{k} s_{k}}\right] \mu_{k}^{*}+\frac{y_{k}^{*} y_{k}^{* T}}{s_{k}{ }^{T} y_{k}{ }^{*}}
$$

where:

$$
\mu_{k}^{*}=s_{k}^{T} y_{k}^{*} / y_{k}^{*} y_{k}^{* T}
$$

and $B_{k}$ satisfies the secant condition as follows:

$$
B_{k+1} s_{k}=\mu_{k}^{*} y_{k}^{*}
$$

outline of the new non-monotone self-scaling MBFGS described in Algorithm 1.

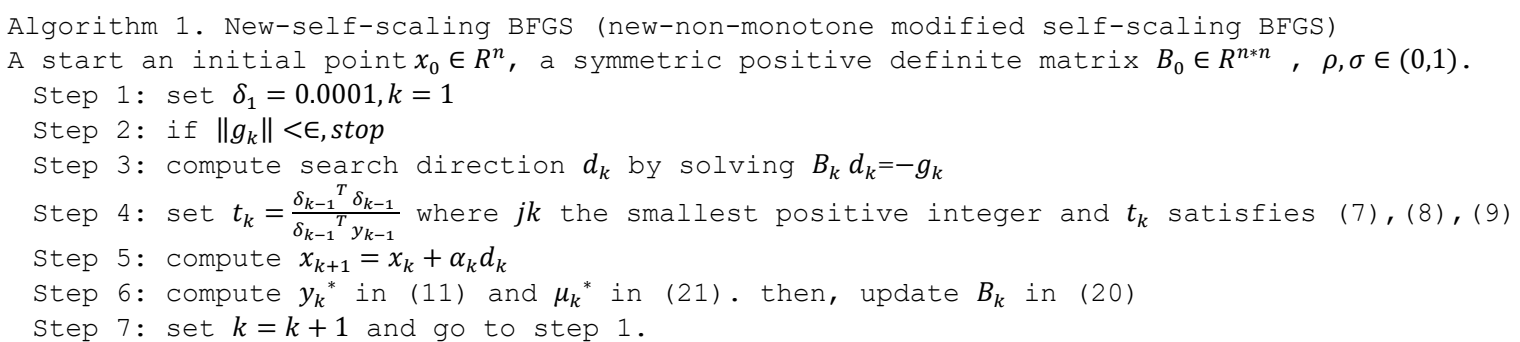

\section{CONVERGENCE ANALYSIS}

For the general nonlinear objective function, this part is to explain and prove the properties of the new algorithm. And the following assumptions on the objective function (f).

\subsection{Assumption (H)}

The level set $S=\left\{x: x \in R^{n}, f(x) \leq f\left(x_{1}\right)\right\}$ is bounded, where $x_{1}$ is the starting point. In a neighborhood $\Omega$ of $S$, $\mathrm{f}$ is continuously differentiable and its gradient $\mathrm{g}$ is Lipchitz continuously, namely, there exists a constant $L \geq 0$ such that $\left\|g(x)-g\left(x_{k}\right)\right\| \leq L\left\|x-x_{k}\right\|, \forall x, x_{k} \in \Omega$. It is clear that from the assumption $(\mathrm{H}, \mathrm{i})$, there exists a positive constant $\mathrm{D}$ such that $D=\max \left\{\left\|x-x_{k}\right\| \forall x, x_{k} \in S\right\}$.

\subsection{Some related propertis}

Some proven mathematical properties to completing the stability study of the theoretical side. Property (1). Let $\left\{x_{k}\right\}$ is the sequence generated by Algorithm 1 new-non-monotone self-scaling MBFGS, then $\left\{E_{k}\right\}$ is a non-increasing sequence and for all $k \in N \cup\{0\},\left\{x_{k}\right\} \subset S\left(x_{0}\right)$. Proof: See [22]. Property (2). If the assumptions $(\mathrm{H}, \mathrm{i})$ and $(\mathrm{H}, \mathrm{ii})$ are contented and $\left\{x_{k}\right\}$ is the sequence produced by the new Algorithm 1 (new-non-self-scaling MBFGS). If $\left\|g_{k}\right\| \geq \zeta$ holds for all $k \in N$ with a constant $\zeta>0$ then there exist positive constants $\theta_{1}, \theta_{2}, \theta_{3}$ such that, for all $k \in N$, the inequalities:

$$
\left\|B_{i} s_{i}\right\| \leq \theta_{1}\left\|s_{i}\right\|, \theta_{2}\left\|s_{i}\right\|^{2} \leq s_{i}^{2} B_{i} s_{i} \leq \theta_{3}\left\|s_{i}\right\|^{2}
$$

contract for fully a half of the indices $i \in\{1,2, \ldots \ldots, k\}$.

Proof: To prove that, must offer that there subsist two positive $r$ and $\mathrm{R}$ such that:

$$
\frac{y_{k}^{* T} S_{k}}{\left\|S_{k}\right\|^{2}} \geq r
$$

and

$$
\frac{\left\|y_{k}{ }^{*}\right\|^{2}}{y_{k}{ }^{* T} S_{k}} \leq R
$$

From assumption $\left\|g_{k}\right\| \geq \zeta$ and from (17) we have: 


$$
y_{k}^{* T} s_{k} \geq\left\|g_{k}\right\|^{\beta}\left\|y_{k}{ }^{*}\right\|^{2} \geq \zeta^{\beta}\left\|y_{k}{ }^{*}\right\|^{2} \geq \zeta^{\beta} \gamma^{\dot{\nu}}\left\|s_{k}\right\|^{2}
$$

so: $\frac{y_{k}{ }^{* T} S_{k}}{\left\|S_{k}\right\|^{2}} \geq r$, where $r=\zeta^{\beta} \gamma^{\dot{\sim}}$ is a positive constant. On the other hand, it follows (11), (12) and CauchySchwartz inequality that:

$$
\left\|y_{k}^{*}\right\| \leq\left\|y_{k}\right\|+\left\|s_{k}\right\|\left(\left\|g_{k}\right\|^{\mu}+\frac{\left\|y_{k}\right\|}{\left\|s_{k}\right\|}\right)
$$

and from assumptions $(\mathrm{H}, \mathrm{i}),(\mathrm{H}, \mathrm{ii})$ and the relation in corollary (3.3) there exists $\hat{R}>0$ such that $\left\|g_{k}\right\| \leq \hat{R}$. Therefore, it can be seen that:

$$
\left\|y_{k}^{*}\right\| \leq\left\|s_{k}\right\|\left(L+\hat{R}^{\mu}+L\right)=C\left\|s_{k}\right\|
$$

$\mathrm{L}$ is Lipchitz constant from a hypothesis $(\mathrm{H}, \mathrm{ii})$, and $C=L+\hat{R}^{\mu}+L$. The relation (26) along with (27) for all $k \in N$, result:

$$
\frac{\left\|y_{k}^{*}\right\|^{2}}{y_{k}^{* T} S_{k}} \leq R
$$

where: $\mathrm{R}=\frac{c^{2}}{\zeta^{\mu}}$. From (24), (25), and theorem (2.1) in [6] we have the rest of the proof.

Property (3). If the assumption $(\mathrm{H}, \mathrm{i})$ and (H, ii) exist and $\left\{x_{k}\right\}$ is the sequence generated by the New1 algorithm. If $\left\|g_{k}\right\| \geq \zeta$ holds for all $k \in N$ with a constant $\zeta>0$ then there is a positive constant $\breve{t}$ such that $t_{k}>t$ for all $\mathrm{k}$ belonging to $\mathrm{J}=\{k \in N$ hold (16) $\}$. Proof: see [22]. Property (4). Suppose that the assumption $(\mathrm{H}, \mathrm{i})$ and $(\mathrm{H}, \mathrm{ii})$ hold, then:

$$
\sum_{k=0}^{\infty}-\alpha_{k} g_{k}{ }^{T} d_{k}<\infty
$$

Proof: Using (7), (8), (9) we have:

$$
f_{k+1}-f_{k} \leq \sigma \alpha_{k} g_{k}{ }^{T} d_{k}=-\sigma \alpha_{k}\left(\left\|g_{k+1}\right\|^{2}+\left[1+\frac{\left\|y_{k}{ }^{*}\right\|^{2}}{y_{k}{ }^{*} S_{k}}\right] \frac{\left(s_{k}{ }^{T} g_{k+1}\right)^{2}}{y_{k}{ }^{* T} S_{k}}\right) \leq 0
$$

therefore, $\left\{f_{k}\right\}$ is a decreasing sequence. Since $\mathrm{f}$ is bounded below, there exists a constant $\hat{f}$ such that:

$\lim _{k \rightarrow \infty} f_{k}=\hat{f}$. It follows that: $\sum_{k=0}^{\infty}\left(f_{k}-f_{k+1}\right)=\lim _{\sigma \rightarrow \infty} \sum_{k=0}^{\sigma}\left(f_{k}-f_{k+1}\right)=\lim _{\sigma \rightarrow \infty}\left(f_{0}-f_{\sigma+1}\right)=f_{0}-\hat{f}$ Hence, $\sum_{k=0}^{\infty}\left(f_{k}-f_{k+1}\right)<+\infty$.

\subsection{Theorem}

If the assumption $(\mathrm{H}, \mathrm{i})$ and $(\mathrm{H}, \mathrm{ii})$ exist and $\left\{x_{k}\right\}$ is the sequence generated by the New Algorithm 1 (self-scaling NBFGS), then:

$$
\lim _{k \rightarrow \infty} \text { in } f\left\|g_{k}\right\|=0 .
$$

Proof: If we assume that $\lim _{k \rightarrow \infty}$ in $f\left\|g_{k}\right\| \neq 0$, so there exists a constant $\zeta>0$ such that $\left\|g_{k}\right\| \geq \zeta$.For all $\mathrm{k}$ sufficiently, since $\mathrm{B}_{\mathrm{k}} \mathrm{s}_{\mathrm{k}}=\alpha_{\mathrm{k}} \mathrm{B}_{\mathrm{k}} \mathrm{d}_{\mathrm{k}}=-\alpha_{\mathrm{k}} g_{\mathrm{k}}$, it follows from (28) that $\sum_{k=0}^{\infty} \alpha_{k} \frac{s_{k}{ }^{T} \mathrm{~B}_{\mathrm{k}} \mathrm{s}_{\mathrm{k}}}{\left\|\mathrm{B}_{\mathrm{k}} \mathrm{s}_{\mathrm{k}}\right\|}\left\|g_{k}\right\|^{2}=$ $\sum_{k=0}^{\infty} \frac{1}{\alpha_{k}} s_{k}{ }^{T} \mathrm{~B}_{\mathrm{k}} \mathrm{s}_{\mathrm{k}}=\sum_{k=0}^{\infty}\left(-\alpha_{k} g_{\mathrm{k}}{ }^{T} \mathrm{~d}_{\mathrm{k}}\right)<\infty .\left\|g_{k}\right\| \geq \zeta$, From the property (3) definition of J are holds, leads us to:

$$
\begin{aligned}
& \sum_{k=0}^{\infty} \alpha_{k} \frac{S_{k} \mathrm{~B}_{\mathrm{k}} S_{\mathrm{k}}}{\left\|\mathrm{B}_{\mathrm{k}} S_{\mathrm{k}}\right\|^{2}}\left\|g_{k}\right\|^{2} \geq \zeta^{2} \sum_{k=0}^{\infty} \alpha_{k} \frac{S_{k}{ }^{T} \mathrm{~B}_{\mathrm{k}} S_{\mathrm{k}}}{\left\|\mathrm{B}_{\mathrm{k}} \mathrm{S}_{\mathrm{k}}\right\|^{2}} \\
& \geq \zeta^{2} \sum_{k \in J}^{\infty} \alpha_{k} \frac{S_{k}{ }^{T} \mathrm{~B}_{\mathrm{k}} \mathrm{S}_{\mathrm{k}}}{\left\|\mathrm{B}_{\mathrm{k}} \mathrm{S}_{\mathrm{K}}\right\|^{2}} \\
& >\zeta^{2} \bar{\alpha} \sum_{k \in J}^{\infty} \frac{S_{k} \mathrm{~B}_{\mathrm{k}} S_{\mathrm{k}}}{\left\|\mathrm{B}_{\mathrm{k}} \mathrm{S}_{\mathrm{k}}\right\|^{2}}
\end{aligned}
$$

from the last inequity in which comes from property (4) this leads to: 


$$
\sum_{k \in J}^{\infty} \frac{S_{k}^{T} \mathrm{~B}_{\mathrm{K}} S_{\mathrm{k}}}{\left\|\mathrm{B}_{\mathrm{k}} S_{\mathrm{k}}\right\|^{2}}<\infty
$$

because the set $\mathrm{J}$ is infinite, it is lead to that $\frac{s_{k}{ }^{T} \mathrm{~B}_{\mathrm{K}} \mathrm{s}_{\mathrm{k}}}{\left\|\mathrm{B}_{\mathrm{k}} \mathrm{s}_{\mathrm{k}}\right\|^{2}} \rightarrow 0$ for $k \in J$.This immediately contradicts the fact: $\frac{s_{k}{ }^{T} B_{\mathrm{K}} s_{\mathrm{K}}}{\left\|\mathrm{B}_{\mathrm{K}} S_{\mathrm{K}}\right\|^{2}} \geq \frac{\vartheta_{2}\left\|s_{k}\right\|^{2}}{\vartheta_{1}^{2}\left\|s_{k}\right\|^{2}}=\frac{\vartheta_{2}}{\vartheta_{1}^{2}}$ that is in (31).

\section{RESULTS AND DISCUSSION}

The main work of this section is to compare the numerical experiments of the New1 non-monotone modified MBFGS algorithm with the (MBFGS-XG) algorithm proposed by [23]. We present a new algorithm in which the new non-monotone line search to approximate comparison is named (New1 NMBFGS). On the other hand, we compare the numerical experiments of the new self-scaling modified BFGS algorithm named (New2 self-scaling MBFGS) with the standard self-scaling BFGS method straight with Armijo line search [7], [9]. We wrote FORTRAN language and double-precision arithmetic. These results were performed on a PC. Our attempts were performed onset of (50) nonlinear unconstrained problems that have a second derivative available, and the experience problems are contributed in CUTE [24], [25].

We considered numerical experiments with several variable $n=2,4,6, \ldots \ldots 1000$, All these methods terminate when the following stopping criterion is met $\left\|g_{k}\right\|_{\infty} \leq 10^{-6}$. Our experiences show the parameters $\rho=0.46, \sigma=0.38, \delta_{1}=0.0001$, have the best conclusions for all the algorithms. Tables 1 and 2 compare some numerical experiments for the New1, New2 of algorithms against the BFGS algorithms, and the test problems with different dimensions, $n=2,4, \ldots 1000$. In all these tables: $\mathrm{N}=$ Dimension of the problem, $\mathrm{NOI}=$ number of iterations, $\mathrm{NOF}=$ Number of functions, $\mathrm{CPU}=$ Total time required to complete the evaluation process for each test problem.

Figures 1 to 4 compare of the New1 method against MBFGS-XG method due to NOI and it's clear that New1 have more than $37.89 \%$, and $66.71 \%$ NOI, and New2 against self-scaling BFGS due to NOI and New2 have more than $44.18 \%$ and $70.76 \%$ NOI respectively. Also Figures 2 and 5 compares the New1 against MBFGS-XG method $38.28 \%$ and $44.27 \%$ due to NOF, and New2 against self-scaling BFGS due to NOF and it's better than $42.29 \%$ and $69.2 \%$ respectively. Figures 3 and 6 compares of the New1 method against MBFGS-XG and have better results in comparison $70.7 \%$ and $71 \%$, and New2 against self-scaling BFGS $70.41 \%$ and $70.7 \%$ due to CPU [26]-[28].

Table 1. Comparison of the New1 method against MBFGS-XG and New2 against self-scaling BFGS method

\begin{tabular}{|c|c|c|c|c|c|c|c|c|c|c|c|c|}
\hline \multirow{2}{*}{ Prob. } & \multicolumn{3}{|c|}{ MBFGS-XG method } & \multicolumn{3}{|c|}{ New1 Method } & \multicolumn{3}{|c|}{ Self-scaling BFGS method } & \multicolumn{3}{|c|}{ New2 Method } \\
\hline & NOI & NOF & CPU & NOI & NOF & $\mathrm{CPU}$ & NOI & NOF & CPU & NOI & $\mathrm{NOF}$ & CPU \\
\hline 1 & 15 & 35 & 2.2 & 12 & 35 & 2.29 & 11 & 42 & 2.1 & 11 & 42 & 2.91 \\
\hline 2 & 9 & 19 & 0.02 & 9 & 19 & 0.01 & 9 & 19 & 0.01 & 9 & 19 & 0.01 \\
\hline 3 & 44 & 64 & 0.3 & 33 & 58 & 0.05 & 36 & 70 & 0.05 & 29 & 72 & 0.05 \\
\hline 4 & 15 & 35 & 0.19 & 10 & 31 & 0.01 & 15 & 35 & 0.9 & 10 & 31 & 0.01 \\
\hline 5 & 21 & 26 & 0.45 & 20 & 30 & 0.21 & 25 & 30 & 0.01 & 8 & 19 & 0.21 \\
\hline 6 & 13 & 28 & 1.91 & 13 & 41 & 0.1 & 12 & 53 & 1.9 & 16 & 69 & 0.1 \\
\hline 7 & 39 & 55 & 2.5 & 41 & 59 & 2.5 & 38 & 62 & 2.3 & 41 & 59 & 2.5 \\
\hline 8 & 10 & 25 & 1.2 & 7 & 22 & 0.13 & 7 & 22 & 1.2 & 7 & 22 & 0.13 \\
\hline 9 & 80 & 113 & 3.1 & 75 & 110 & 2.9 & 70 & 102 & 2.3 & 75 & 110 & 2.9 \\
\hline 10 & 25 & 60 & 2.5 & 22 & 47 & 0.5 & 25 & 70 & 2.4 & 22 & 47 & 0.5 \\
\hline 11 & 1310 & 8600 & 0.3 & 401 & 3001 & 0.1 & 640 & 5299 & 0.2 & 140 & 1109 & 0.1 \\
\hline 12 & 101 & 621 & 0.32 & 70 & 551 & 0.0 & 70 & 501 & 0.2 & 35 & 305 & 0.0 \\
\hline 13 & 80 & 502 & 0.1 & 51 & 320 & 0.1 & 60 & 402 & 0.1 & 23 & 349 & 0.1 \\
\hline 14 & 800 & 3001 & 1.6 & 75 & 300 & 0.1 & 80 & 341 & 1.5 & 30 & 150 & 0.01 \\
\hline 15 & 291 & 1100 & 0.91 & 60 & 250 & 0.01 & 54 & 245 & 0.61 & 23 & 120 & 0.01 \\
\hline 16 & 1372 & 8911 & 2.9 & 401 & 3221 & 2.9 & 640 & 5320 & 2.1 & 139 & 1001 & 2.9 \\
\hline 17 & 1050 & 7000 & 0.04 & 200 & 972 & 0.1 & 200 & 1608 & 0.04 & 110 & 890 & 0.01 \\
\hline 18 & 180 & 181 & 0.41 & 180 & 181 & 0.41 & 180 & 181 & 0.41 & 180 & 181 & 0.41 \\
\hline 19 & 1340 & 9101 & 2.91 & 604 & 2952 & 2.91 & 1291 & 8517 & 2.1 & 207 & 1201 & 2.03 \\
\hline 20 & 1311 & 8500 & 0.015 & 520 & 3970 & 0.015 & 1341 & 8500 & 0.015 & 170 & 1418 & 0 \\
\hline 21 & 589 & 479 & 0.12 & 431 & 530 & 0.12 & 589 & 749 & 0.12 & 322 & 419 & 0.12 \\
\hline 22 & 220 & 1601 & 0.639 & 22 & 380 & 0.639 & 23 & 204 & 0.639 & 16 & 190 & 0.639 \\
\hline 23 & 150 & 891 & 0.46 & 45 & 300 & 0.0 & 21 & 185 & 0.46 & 17 & 140 & 0.0 \\
\hline 24 & 299 & 1297 & 0.15 & 90 & 499 & 0.01 & 94 & 610 & 0.15 & 40 & 301 & 0.01 \\
\hline 25 & 470 & 1992 & 0.0 & 122 & 601 & 0.0 & 130 & 75 & 0.0 & 115 & 537 & 0.0 \\
\hline Total & 9834 & 54237 & 25.244 & 3514 & 18488 & 16.114 & 5661 & 33242 & 21.814 & 1796 & 8825 & 15.659 \\
\hline
\end{tabular}


Table 2. Comparison of the New1 method against MBFGS-XG and New2 against self-scaling BFGS method

\begin{tabular}{|c|c|c|c|c|c|c|c|c|c|c|c|c|}
\hline \multirow{2}{*}{ Prob. } & \multicolumn{3}{|c|}{ MBFGS-XG method } & \multicolumn{3}{|c|}{ New1 Method } & \multicolumn{3}{|c|}{ Self-scaling BFGS method } & \multicolumn{3}{|c|}{ New2 Method } \\
\hline & NOI & NOF & $\mathrm{CPU}$ & NOI & & NOI & NOF & $\mathrm{CPU}$ & NOI & & NOI & $\mathrm{NOF}$ \\
\hline 26 & 181 & 391 & 1.19 & 47 & 200 & 0.1 & 50 & 241 & 1.19 & 21 & 149 & 0.1 \\
\hline 27 & 200 & 500 & 1.19 & 65 & 210 & 0.1 & 68 & 250 & 1.19 & 51 & 190 & 0.1 \\
\hline 28 & 245 & 821 & 0.46 & 37 & 126 & 0.46 & 28 & 105 & 0.46 & 19 & 73 & 0.46 \\
\hline 29 & 151 & 391 & 0.05 & 10 & 59 & 0.05 & 13 & 61 & 0.05 & 7 & 50 & 0.05 \\
\hline 30 & 681 & 1501 & 0.21 & 520 & 1101 & 0.21 & 579 & 1391 & 0.21 & 434 & 1031 & 0.21 \\
\hline 31 & 780 & 8001 & 0.0 & 121 & 781 & 0.1 & 124 & 690 & 0.0 & 112 & 583 & 0.1 \\
\hline 32 & 981 & 6351 & 0.25 & 392 & 4381 & 0.25 & 428 & 4221 & 0.15 & 219 & 4001 & 0.25 \\
\hline 33 & 100 & 520 & 1.24 & 15 & 40 & 0.91 & 15 & 40 & 1.9 & 15 & 40 & 0.91 \\
\hline 34 & 180 & 690 & 0.0 & 20 & 69 & 0.0 & 29 & 119 & 0.0 & 18 & 59 & 0.0 \\
\hline 35 & 1050 & 7211 & 1.1 & 189 & 1481 & 1.19 & 190 & 1591 & 1.1 & 99 & 790 & 1.23 \\
\hline 36 & 520 & 1300 & 0.9 & 79 & 245 & 1.31 & 43 & 159 & 0.5 & 25 & 80 & 1.51 \\
\hline 37 & 325 & 981 & 0.05 & 56 & 105 & 0.01 & 28 & 160 & 0.01 & 9 & 49 & 0.01 \\
\hline 38 & 200 & 1231 & 0.35 & 117 & 963 & 0.35 & 187 & 1141 & 0.15 & 133 & 1121 & 0.35 \\
\hline 39 & 17 & 18 & 0.15 & 17 & 18 & 0.18 & 17 & 18 & 0.15 & 17 & 18 & 0.18 \\
\hline 40 & 19 & 31 & 0.01 & 19 & 31 & 0.01 & 19 & 31 & 0.01 & 19 & 31 & 0.01 \\
\hline 41 & 85 & 791 & 0.9 & 60 & 572 & 0.95 & 71 & 613 & 0.01 & 50 & 495 & 0.95 \\
\hline 42 & 35 & 69 & 0.8 & 35 & 77 & 0.0 & 32 & 64 & 0.02 & 35 & 77 & 0.0 \\
\hline 43 & 35 & 78 & 1.9 & 20 & 70 & 1.9 & 30 & 70 & 1.9 & 15 & 60 & 1.9 \\
\hline 44 & 172 & 4832 & 0.9 & 60 & 840 & 0.0 & 121 & 1960 & 0.5 & 45 & 840 & 0.0 \\
\hline 45 & 101 & 200 & 0.63 & 139 & 215 & 1.5 & 101 & 200 & 0.61 & 139 & 215 & 1.5 \\
\hline 46 & 19 & 30 & 1.5 & 11 & 27 & 1.6 & 16 & 27 & 0.39 & 10 & 20 & 1.6 \\
\hline 47 & 176 & 1981 & 0.01 & 142 & 1210 & 0.01 & 170 & 1900 & 0.01 & 89 & 982 & 0.01 \\
\hline 48 & 69 & 121 & 0.81 & 41 & 121 & 0.6 & 50 & 129 & 0.05 & 39 & 117 & 0.6 \\
\hline 49 & 37 & 77 & 0.15 & 21 & 70 & 0.12 & 30 & 70 & 0.01 & 21 & 67 & 0.12 \\
\hline 50 & 30 & 50 & 0.01 & 29 & 50 & 0.01 & 25 & 44 & 0.01 & 26 & 49 & 0.01 \\
\hline Total & 6389 & 38167 & 14.76 & 2262 & 13062 & 11.92 & 2464 & 15295 & 10.58 & 1667 & 11187 & 12.16 \\
\hline
\end{tabular}

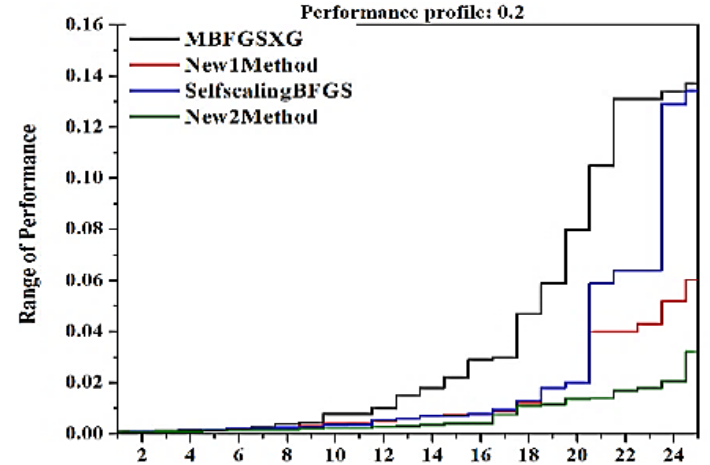

Figure 1. Performance due to NOI NOF CPU

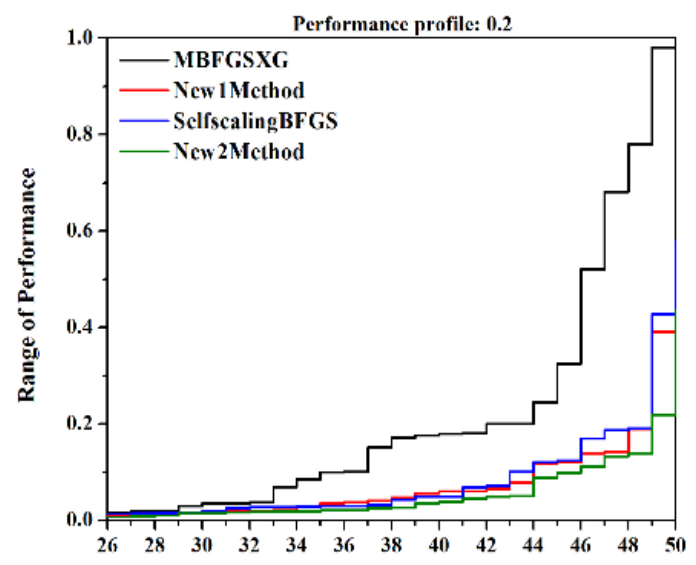

Figure 3. Performance due to NOI NOF CPU

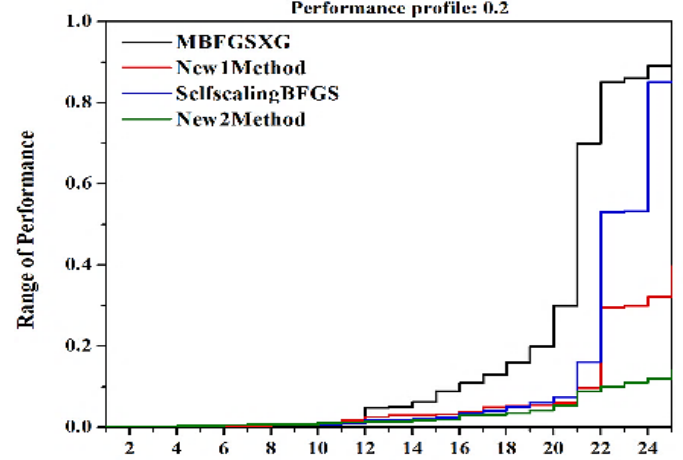

Figure 2. Performance due to NOI NOF CPU

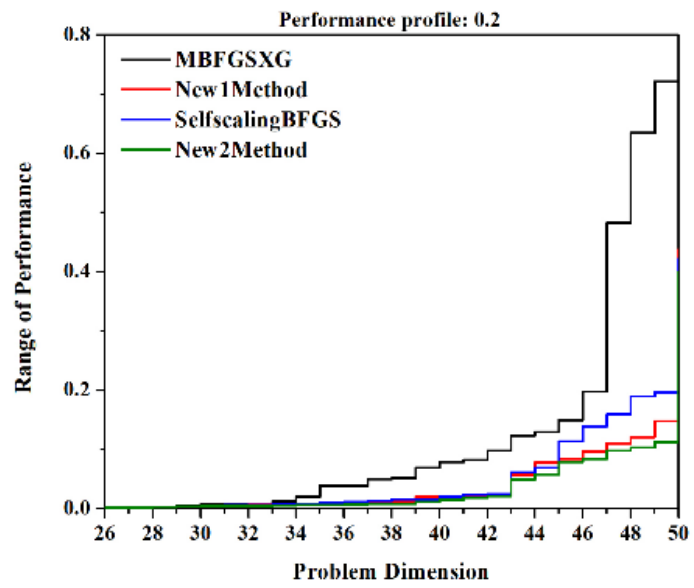

Figure 4. Performance due to NOI NOF CPU 


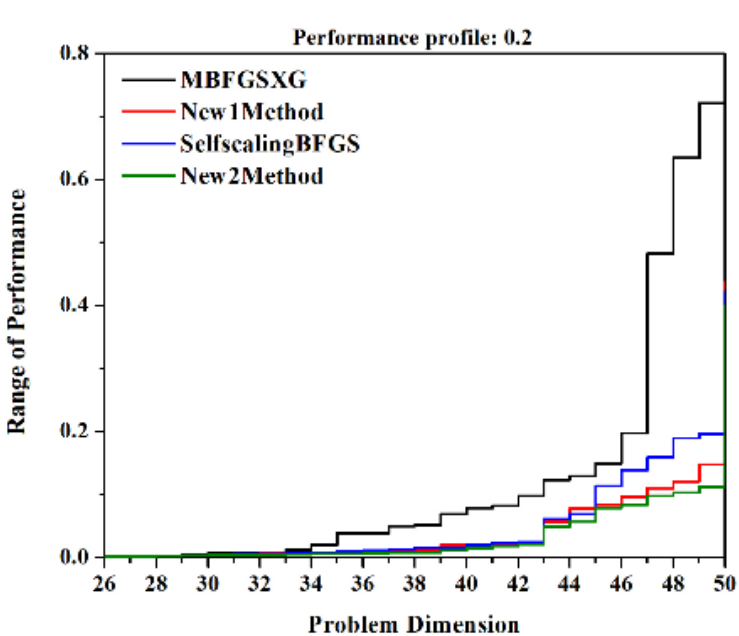

Figure 5. Performance due to NOI NOF CPU

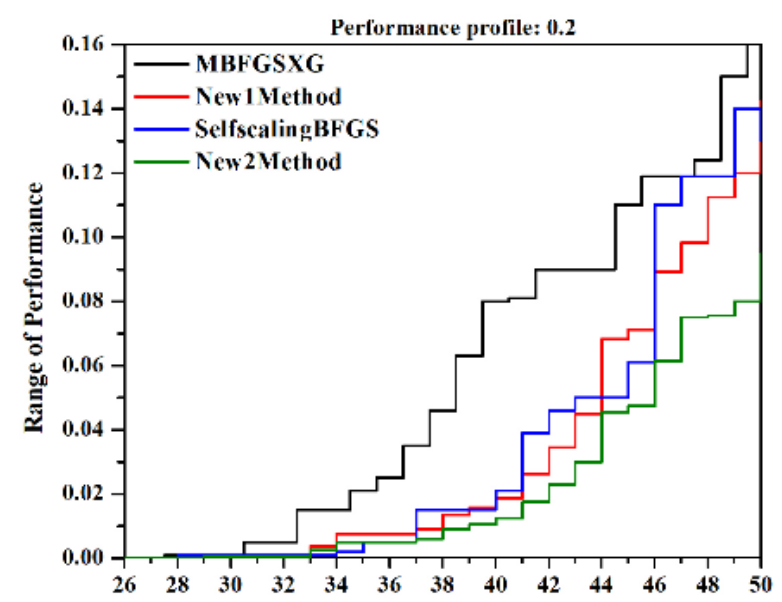

Figure 6. Performance due to NOI NOF CPU

\section{CONCLUSION}

In this paper, we have proposed a new non-monotone BFGS algorithm and combined it with a new modified self-scaling BFGS update to a sacrificial Hessian matrix with a known line search planning for nonconvex optimization problems. It is clear that a new non-monotone can progress the probability of finding a global optimum and also promote speed of convergence especially in presence of a narrow-curved valley and sufficient descent property of algorithm convergence. Thus, in our algorithms, we are enjoyable to get benefits from their properties. Lastly, our numerical results show that our new algorithms have competitive with the standard self-scaling BFGS method and have robust numerical results as compared to the nonmonotone (self-scaling BFGS) algorithm had proposed.

\section{ACKNOWLEDGEMENTS}

The research is supported by the College of Computer Sciences and Mathematics, University of Mosul, Republic of Iraq. The author declares that there are no conflicts of interest regarding this work.

\section{REFERENCES}

[1] P. I. Toint, "An assessment of non-monotone linear search technique for unconstrained optimization," SIAM Journal on Scientific Computing, vol. 17, no. 3, pp. 725-739, 1996, doi: 10.1137/S106482759427021X.

[2] S. S. Oren, "Self-Scaling Variable Metric (SSVM) Algorithms. Part II: Implementation and Experiments," Management Science, vol. 20, no. 5, pp. 862-874, 1974.

[3] S. S. Oren and D. G. Lunerberger, "Self-Scaling Variable Metric algorithm. Part I: Criteria and Sufficient Conditions for Scaling a Class of Algorithms," Management Science, vol. 20, no. 5, pp. 845-862, 1974.

[4] D.-H. Li and M. Fukushima, "A modified BFGS method and its global convergence in non-convex minimization," Journal of Computational and Applied Mathematics, vol. 129, no. 1-2, pp. 15-35, 2001, doi: 10.1016/S0377-0427(00)00540-9.

[5] L. Zhang, and J. Li, "A new globalization technique for nonlinear conjugate gradient methods for no convex minimization," Applied Mathematics and computation, vol. 217, no. 24, pp. 10295-10304, 2011, doi: 10.1016/j.amc.2011.05.032.

[6] M. J. D. Powell, "Some global convergence properties of a variable metric algorithm for minimization without exact line searches," Nonlinear programming, Slam-AMS Proceedings, vol. 9, pp. 53-72, 1976.

[7] J. J. More, B. S. Garbow, and K. E. Hillstrom, "Testing Unconstrained optimization software," ACM Transactions on Mathematical Software, vol. 7, no. 1, pp. 17-41, 1981, doi: 10.1145/355934.355936.

[8] G. Liu, J. Han, and D. Sun, "Global convergece of the bfgs algorithm with nonmonotone linesearch *this work is supported by national natural science foundation\$ef," A Journal of Mathematical Programming and Operations Research, vol. 34, no. 2, pp. 147-159, 1995, doi: 10.1080/02331939508844101.

[9] L. Liu, S. Yao, and Z. Wei, "The global and superlinear convergence of a new non-monotone MBFGS algorithm on convex objective function," Journal of Computational and Applied Mathematics, vol. 220, no. 1-2, pp. 422-438, 2008, doi: 10.1016/j.cam.2007.08.017.

[10] Y. Xio, H. Sun, and Z. Wang, "A globally convergent BFGS method with non-monotone line search for nonconvex minimization," Journal of Computational and Applied Mathematics, vol. 230, no. 1, pp. 95-106, 2009, doi: 10.1016/j.cam.2008.10.065. 
[11] H. C. Zhang and W. W. Hager, "A non-monotone line search technique and its application to unconstrained optimization," SIAM Journal on Optimization, vol. 14, no. 4, pp. 1043-1056, 2004, doi: $10.1137 / \mathrm{S} 1052623403428208$.

[12] D. H. Li and M. Fukushima, "On Global convergence of the BFGS method for non-convex unconstrained optimization problems," SIAM Journal on Optimization, vol. 11, no. 4, pp. 1054-1064, 2001, doi: $10.1137 /$ S1052623499354242.

[13] Y. H. Dai, "On the non-monotone line search," Journal of Optimization Theory and Applications, vol. 112, no. 2, pp. 315-330, 2002, doi: 10.1023/A:1013653923062.

[14] I. Bongartz, A. R. Conn, N. I. M. Gloud, and P. L. Toint, "CUTE: Constrained and Unconstrained Testing Environment," ACM Transactions on Mathematical Software, vol. 21, no. 1, pp. 123-160, 1995, doi: $10.1145 / 200979.201043$.

[15] W. F. Mascarenhas, "The BFGS method with exact line searches fails for non-convex objective functions," Mathematical Programming, vol. 99, pp. 49-61, 2004, doi: 10.1007/s10107-003-0421-7.

[16] M. Miladinovic, P. Stanimirovic, and S. Miljkovic, "Scalar correction method for solving large scale unconstrained Minimization Problems," Journal of Optimization Theory and Applications, vol. 151, pp. 304-320, 2011, doi: 10.1007/s10957-011-9864-9.

[17] G. E. Manoussakis, D. G. Sotiropoulus, C. A. Botsaris, and T. N. Grapsa, "A non-monotone Conic Method for Unconstrained Optimization," in Proceedings of 4th GRACM, Congress on Computational Mechanics, 2002, pp. 27-29.

[18] J. Nocedal and S. J. Wright, "Numerical Optimization,” Springer, 2006, doi: 10.1007/978-0-387-40065-5.

[19] M. A1-Balli and H. Khalfan, "A combined class of self-scaling and modified Quasi-Newton methods," Computational Optimization and Applications, vol. 52, no. 2, pp. 393-408, 2012, doi: 10.1007/s10589-011-9415-1.

[20] K. Amini, S. Bahrami and S. Amiri, "A Non-monotone Modified BFGS Algorithm for Non-convex Unconstrained Optimization problems," Filomat, vol. 30, no. 5, pp. 1283-1296, 2016, doi: 10.2298/FIL1605283A.

[21] G. Chao and Z. Detong, "A non-monotone line search filter method with reduced Hessian updating for nonlinear optimization," Journal of Systems Science and Complexity, vol. 26, pp. 534-555, 2013, doi: 10.1007/s11424-012-0036-2.

[22] Y. Z. Yuan, "A modified BFGS algorithm for unconstrained optimization," IMA Journal of Numerical Analysis, vol. 11, no. 3, pp. 325-332, 1991, doi: 10.1093/imanum/11.3.325.

[23] J. Zhang, Y. Xiao, and Z. Wei, "Nonlinear Conjugate gradient methods with sufficient descent condition for largescale unconstrained optimization," Mathematical Problems in Engineering, vol. 2009, pp. 1-16, 2009, doi: $10.1155 / 2009 / 243290$.

[24] R. Z. Al-Kawaz, A. Y. Al Bayati, and M. Jameel, "Interaction between un-updated FR-CG algorithms with an optimal Cuckoo algorithm," Indonesian Journal of Electrical Engineering and Computer Science (IJEECS), vol. 19, no. 3, pp. 1497-1504, 2020, doi: 10.11591/ijeecs.v19.i3.pp1497-1504.

[25] A. Y. Al-Bayati and M. S. Jameel, "New Scaled Proposed Formulas for Conjugate Gradient Methods in Unconstrained Optimization," AL-Rafidain Journal of Computer Sciences and Mathematics, vol. 11, no. 2, pp. 25-46, 2014, doi: 10.33899/csmj.2014.163748.

[26] S. Huang and Z. Wan, "A new nonmonotone spectral residual method for nonsmooth nonlinear equations," Journal of computation and applied mathematics, vol. 313, no. C, pp. 82-101, 2017, doi: 10.1016/j.cam.2016.09.014.

[27] A. Z. M. Sofi, M. Mamat, I. Mohd, and B. S. Putra, "An Improved BFGS Search Direction using Exact Line Search for Solving Unconstrained Optimization Problems, Applied Mathematical Sciences, vol 7, no. 2, pp. 73-85, 2013, doi: 10.12988/AMS.2013.13007.

[28] A. Y. Al-Bayati and M. M. M. Ali, "New multi-step three-term conjugate gradient algorithms with inexact line searchs," Indonesian Journal of Electrical Engineering and Computer Science (IJEECS), vol. 19, no. 3, pp. 1564-1573, 2020, doi: 10.11591/ijeecs.v19.i3.pp1564-1573.

\section{BIOGRAPHY OF AUTHOR}

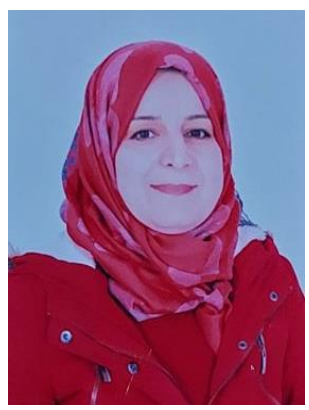

Muna M. M. Ali, Teaching in the Department of Mathematics, College of Computers Sciences and Mathematics, Mosul University, Al-Majmoaa Street, Mosul, Iraq. I completed my $\mathrm{PhD}$ in Numerical optimization. I have 12 national and international published jont and single research papers. Email: munamoh74@uomosul.edu.iq 